\title{
THE FATE OF BINARY SYSTEMS AFTER THE EXPLOSION OF SNE 1993J AND 1994I
}

\author{
HITOSHI YAMAOKA \\ Department of Physics, Faculty of Science, Kyushu University, \\ 4-2-1 Ropponmatsu, Chuo-ku, Fukuoka 810, Japan
}

\begin{abstract}
The kinematics of the binary systems to which supernovae(SNe) 1993J and 1994I belonged are studied. The kick velocity caused by supernova(SN) asymmetry will highly influence the fate of the system. The survival rates with assumed kick velocity distribution are discussed.
\end{abstract}

\section{Introduction}

The progenitors of type Ib/Ic/IIb SNe are considered to be massive stars in close binary systems, of which the outer envelope are torn off. Because of large mass ejection, $\mathrm{SN}$ in the binary will destroy the system in most case. But if the SN explosion is asymmetric and/or if mass ejection is small, the binary system can survive. They can be the origin of the Be/X-ray binary or the binary pulsers, and so on.

Type IIb SN 1993J and type Ic SN 1994I are heavyly investigated and the progenitor models are gotten in hand. With these models and appropriate assumptions, we can construct the models of the binary systems.

\section{Models}

The models for the progenitors are taken from Shigeyama et al. (1993) and Nomoto et al. (1994), and their properties are summarized in Table 1. The mass of the produced neutron star are assumed to be $1.36 M_{\odot}$ for both case. For SN 1993J system, the mass transfer should be nearly conservative, so we take $20 M_{\odot}$ star as the companion. Its radius is taken as $6 R_{\odot}$. For SN 1994I system, we consider three cases: the companion is (A) $1.4 M_{\odot}$ neutron star, (B) $1 M_{\odot}$ white dwarf, (C) $1 M_{\odot}$ main sequence star. 
Assumimg that the orbit just before the explosion is circular and the Roche lobe is filled up by either the progenitor or the companion, the orbital radius $a_{0}$ is determined. The SN ejecta which hits the companion will give the kinetic energy to the companion, and the efficiency $\gamma$ is assumed to be unity (see Yamaoka et al., 1992). The kick velocity $v_{k}$ is expected to be isotropic, and $v_{k}^{2}$ are assumed to follow $\chi^{2}$ distribution.

\section{Results and Discussion}

For SN 1993J system, small $v_{k}$ will break the binary for any kick direction, because the orbital velocity before explosion is rather small. The maximum $v_{k}$ for the system to survive is $v_{k, \max } \sim 140 \mathrm{~km} \mathrm{~s}^{-1}$. On the other hand, if the explosion is symmetric (i.e., $v_{k}=0$ ), the system will not be destroyed.

For SN 1994I, case (A) and (B) are similar to the case of SN 1993J. Symmetric explosion will not destroy the system, also. But, because of the large orbital velocity before explosion, $v_{k, \max }$ are very large. Case $(\mathrm{C})$ is quite different from others: the reaction caused by ejecta is quite large, the system cannot be bound with symmetric explosion. The system can survive only if $1100 \mathrm{~km} \mathrm{~s}^{-1}<v_{k}<2150 \mathrm{~km} \mathrm{~s}^{-1}$, but the possibility is very small.

Integrating with the $v_{k}$ distribution, we can derive the "probability of survival" of the binary as the function of mean value of $v_{k}\left(v_{a v e}\right)$, which are taken as $150 \mathrm{~km} \mathrm{~s}^{-1}$ (Lane et al., 1982) and $450 \mathrm{~km} \mathrm{~s}^{-1}$ (Lane and Lorimer, 1994). Results are summarized in Table 1 as $P\left(v_{\text {ave }}\right)$. If $v_{\text {ave }}$ is large, SN 1993J system scarcely survive. Thus, Be/X-ray binary are not likely formed by this scenario. Also, the low-mass X-ray binary can scarcely be formed such the scenario as $1994 \mathrm{I}(\mathrm{C})$.

\begin{tabular}{crrrrrr} 
& $M_{p}$ & $R_{p}$ & $a_{0}$ & $v_{k, \max }$ & $P(150)$ & $P(450)$ \\
\hline SN93J & 4.89 & 300 & 1250 & 140 & 0.118 & 0.006 \\
SN94I(A) & 2.28 & 0.23 & 0.55 & 2520 & 0.995 & 0.752 \\
SN94I(B) & 2.28 & 0.23 & 0.51 & 2410 & 0.986 & 0.700 \\
SN94I(C) & 2.28 & 0.23 & 3.24 & ${ }^{*} 2150$ & $1.8 \mathrm{e}-32$ & $1.4 \mathrm{e}-5$ \\
\hline & $\left(M_{\odot}\right)$ & $\left(R_{\odot}\right)$ & $\left(R_{\odot}\right)$ & $\left(\mathrm{km} \mathrm{s}^{-1}\right)$ & & $*$ See text.
\end{tabular}

Table 1. Summary of inputs and results.

\section{References}

Lyne, A.G., Anderson, B. and Salter, M.J. (1982) MNRAS 201, 503-520.

Lyne, A.G. and Lorimer, D.R. (1994) Nat. 369, 127-129.

Nomoto, K. et al. (1994) Nat. 371, 227-229.

Shigeyama, T. et al. (1994) Ap. J. 420, 341-347.

Yamaoka, H., Shigeyama T. and Nomoto K. (1992) A. Ap. 267, 433-438. 